\title{
La prueba del dulcitol en cepas de Escherichia coli aisladas de infección urinaria y en deposiciones
}

\author{
Dres. G. Zilleruelo*, C. Saieh* y M. Córdova T.M.**.
}

Esta presentación forma parte de una serie de proyectos realizados o en marcha en el Departamento de Nefrourología del Hospital Luis Calvo Mackenna, y cuyo objetivo es estudiar la relación huésped-parásito en las infecciones urinarias a $E$. coli, especialmente en niños con infecciones recurrentes y $\sin$ anomalías detectables en el tracto urinario.

Anteriormente se han analizado las características de la respuesta serológica del huésped frente a la infección urinaria y su posible respuesta de inmunoglobulinas secretadas localmente $^{12}$.

Muchos investigadores han tratado de descubrir cepas nefritogénicas específicas en la infección urinaria con resultados variables ${ }^{3-5}$.

Olling ${ }^{4}$ ha demostrado que las cepas de $E$. coli de tipo más prevalente se presentan en un $65 \%$ en los pacientes con pielonefritis comparado a un $30 \%$ de aparición en las infecciones urinarias asintomáticas o en las deposiciones de niños sanos. Estos últimos pacientes tendrían cepas de $E$. coli de alta sensibilidad y escasa virulencia, como una forma de parasitismo de adaptación al huésped.

Estudios recientes efectuados por Glyn en $1971^{6}$ y Kaijser en $1973^{7}$, han demostrado que el antígeno de envoltura o antígeno $\mathrm{K}$ de la $E$. coli ejercen una acción antifagocítica $\mathrm{e}$ induce inmunotolerancia, y las cepas que lo poseen tienen una virulencia mayor para invadir el tracto urinario. En efecto, las cepas de $E$. coli, ricas en antígeno $\mathbf{K}$, son más capaces de producir compromiso renal en las infecciones urinarias.

\footnotetext{
*Iepto. Nefrourología, Hospital Luis Calvo Mackenna.

**IDepto. Baceteriologia. Hospital Luis Calvo Markenna.
}

Estos resultados han sido interpretados como una predilección especial por el riñón (nefripatogenicidad) de las cepas ricas en antígeno $\mathrm{K}$, más que una virulencia total de estas cepas ${ }^{8}$.

Kalmanson ha demostrado que la presencia de antígeno $\mathrm{K}$ hace más probable que la $E$. coli cause infección renal en ratas, pero una vez ocurrida, la incidencia de cepas $\mathrm{K}(+) 6 \mathrm{~K}(-)$ es igual $^{9}$.

Existen dificultades técnicas en nuestro medio para detectar en forma precisa el antígeno $\mathrm{K}$ a través de la inhibición de la hemaglutinación o bien mediante la inmunoelectroforesis, por lo que se han buscado otros índices de la $E$. coli que se correlacione con el antigeno $\mathrm{K}$.

Kalmanson, ${ }^{9}$ ha encontrado que la capacidad de fermentar el dulcitol se correlaciona bien con la presencia del antígeno $\mathrm{K}$ en cepas de E. coli.

El objetivo de este trabajo fue el de estudiar en forma comparativa la fermentación del dulcitol en 16 cepas de $E$. coli aisladas de los niños con infecciones urinarias recurrentes y 15 cepas de $E$. coli enteropatógena aisladas de deposiciones y 14 cepas de $E$. coli no patógenas de deposiciones usadas como controles.

\section{MATERIAL Y METODO}

Se estudiaron 16 cepas de $E$. coli de infecciones urinarias recurrentes en 16 niños controlados en el Departamento de Nefrourología del Hospital Luis Calvo Mackenna. El diagnóstico de la infección urinaria se hizo mediante el cultivo y recuento de colonias y las pruebas diferenciales descritas para el diagnóstico de esta especie microbiana.

En los 16 niños se estableció además el sitio de la infección mediante la titulación de an- 
ticuerpos séricos anticoli (HA) y la capacidad de concentración máxima de la orina. Se consideró infección urinaria con compromiso renal (pielonefritis aguda), a toda aquélla con título de hemaglutinación 1/64, o bien, osm. urinaria máxima $800 \mathrm{~m}$ osmo/lt. después de una deprivación acuosa de 16 horas.

Se utilizaron como controles 29 cepas de $E$. coli provenientes de deposiciones, las cuales correspondían a 15 E. coli enteropatógenas y 14 a $E$. coli no patógenas.

La fermentación del dulcitol se comprobó a las 72 horas de incubación de un caldo peptonado con rojo fenol y con $1 \%$ de dulcitol. Se consideró positivo aquellos tubos que viraron de amarillo a rojo.

- El diaghóstica de infección urinaria con sompromiso renal fue fundamentado en los 8 niños, especialmente por la capacidad de concentración alterada, ya que los títulos de hemaglutinación no fueron muy elevados y no se hizo curva serológica en estos pacientes.

\section{RESULTADOS}

Se estudiaron 45 cepas, correspondiendo 16 de ellas a Escherichia coli aisladas de niños con infección urinaria, 15 fueron Escherichia colt enteropatógenas y 14 no patógenas aisladas de deposiciones.

Del total de las 16 cepas obtenidas en orina, 9 produjeron pielonefritis aguda, siendo 3 de ellas dulcitol $(+)$ y 6 dulcitol $(-)$, las 7 restantes causaron infección urinaria baja, con 4 cepas dulcitol $(+)$ y las otras 3 fueron dulcitol ( - ). Estas diferencias obtenidas no son estadísticamente significativas.

De las 15 cepas de E.C.EP, 7 fueron dulcitol $(+)$ y 8 dulcitol ( - ).

Las cepas de $E$. coli no patógenas aisladas de deposiciones fueron 14 , siendo 9 del total dulcitol $(+)$ y las otras 5 dulcitol $(-)$. (Véase tabla 1.)

\section{COMENTARIO}

Llama la atención el alto porcentaje de cepas de $E$. coli no patógenas de deposiciones que fermenó el dulcitol (65\%), en tanto que las cepas de $E$. coli enteropatógenas se repartieron en forma equiparada entre el dulcitol $(+)$ y $(-)$.
Tabla 1

PRUEBA DEL. DULCITOL EN 45 (EPAS IDE ESC'HERIC'HIA COLI AISLADAS EN ORINA Y EN DEPOSICIONES

Escherichia coli de infección urinaria: 16 cepas

$$
\begin{aligned}
& \text { Pielonefritis aguda: } \quad 9<\text { Dulcitol }(+) 3 \\
& \text { Infecitol }(-)^{6} \\
& \text { Dulcitol }(+)^{4} \\
& \text { Dulcitol }(-)_{3}
\end{aligned}
$$

Eschertche colı enteropatógena: $\quad 15$ cepas

Dulcitol ( +) 7

Dulcitol ( - ) 8

Exchencha coli no patógena de deposiciones: 14 cepas

Dulcitol ( +$) 9$

Dulcitol (-) 5

En relación a las cepas de $E$. coli de los niños con infección urinaria hay varios hechos destacados. No se observa una clara diferencia en la fermentación del dulcitol entre las cepas de E. coli provenientes de infecciones urinarias altas y bajas; por el contrario, la mayoria de las cepas de pielonefritis eran dulcitol (-). Estos resultados son similares a los obtenidos por Kalmanson. ${ }^{9}$

No se observó correlación entre el grado de compromiso renal y la intensidad de la fermentación del dulcitol.

Llama la atención también la elevada frecuencia de las cepas de $E$. coli de deposiciones, fundamentalmente la no patógena, que resultaron dulcitol $(+)$, lo que indicaría un alto contenido de antígeno $\mathrm{K}$ en las cepas menos virulentas.

Los datos obtenidos en este estudio no nos permiten concluir si existe o no correlación entre la fermentación del dulcitol y la presencia de antígeno $K$, ya que no se hicieron determinaciones de este último antígeno. Sin embargo, parece poco probable que exista la correlación entre virulencia del germen y fermentación del dulcitol sugerida por Kalmanson, ya que como ha demostrado Glyn ${ }^{6}$ la incidencia de cepas de $E$. coli antígeno $\mathrm{K}(+)$ es significativamente superior en las cepas aisladas del tracto urinario superior, respecto a las que se aislan en el tracto inferior $y / o$ en deposiciones, 
hecho que no concuerda con los resultados obtenidos por nosotros, mediante la fermentación del dulcitol.

En resumen, creemos que la fermentación del dulcitol no se correlaciona con la presencia de antígeno $\mathrm{K} \mathrm{y}$, por ende, con la virulencia de la $E$. coli, y, por lo tanto, no es útil en la pesquisa de las cepas que producen compromiso renal en la infección urinaria.

\section{RESUMEN}

Se presentan los resultados obtenidos con la capacidad de fermentar el dulcitol en 45 copas de $E$, coll; 16 cepas provenientes de niños con infección urinaria y 29 cepas aisladas en deposiciones de las cuales 15 eran enteropatógenas y las otras 14 eran no patógenas.

En las cepas de $E$. coli urinarias se obtuwo un $43 \%$ que fermentó el dulcitol a las 72 horas, y la distribución según el sitio de localización de la infección urinaria no reveló diferencias significativas entre las infecciones altas y la fermentación ( +).

Se destaca el alto porcentaje decepas de $E$. coli de deposiciones que fueron dulcitol $(+), 46,6 \%$ la enteropatógena $y$ $64,3 \%$ la cepa no patógena.

Se analizó el significado de la capacidad de fermentar el dulcitol en relación a la presencia de antigeno $K$ en la $E$. colt, y se concluye que es dudoso que exista correlación entre virulencia del germen y fermentación del dulcitol.

\section{SUMMARY}

The result obtained with the fermentation of dulcitol in 45 strains of $E$. coli are presented. Of these, 16 were isolated from urinary tract and 29 were isolated from stools (15 serotyped as enteropathogenic and 14 no pathogenic).

$43,7 \%$ of the urinary strains fermented dulcitol at 72 hours and there was no significative difference between the strains of $E$. coll isolated from lower and from upper urinary tract infections.

A high percentage of $E$. coli fermenting dulcitol was observed in strains from stools; $46 \%$ of the enteropathogenic $E$. colt and $64,3 \%$ of the non pathogenic strains.

The present study suggests that there is no correlation between the presence of antigen $K$ and virulence, therefore. and the fermentation of dulcitol.

\section{REFERENC:IAS}

1 Zillerueto, (i.; Puga, F.; Donoso, E. Rev. Chil. Ped., 44: 563, 1973

2 Zilleruelo, G.; Saieh, C.; Rebolledo, L.; Córdova, M.; Valdés, M.H. Próximo a ser publicado.

3 Vosti, K.L.; Goldberg, L.M.; Monto, A.S.; Rantz, L.A. J. Clin. Invest., 43; 2377, 1964.

- Olling, S.; Hanson, L.A.; Holmgren et al. Infection, $1 ; 24-28 ; 1973$.

3 Veter, E. Pediatr. Clin. North Am., 11; 517,1964

6 Glynn, A.A. et al. Lancet, 1; 514, 1971.

7 Kaijser, B. J. Infect D.S., 127; 670, 1973.

8 Guze, L.B. Yale J. Biol Meal, 48; 203, 1973.

- Kalmanson, G.M.; Harwich, N.J.; Turck, M.; Guze, L.B. Lancet, 18; 136, 1975. 